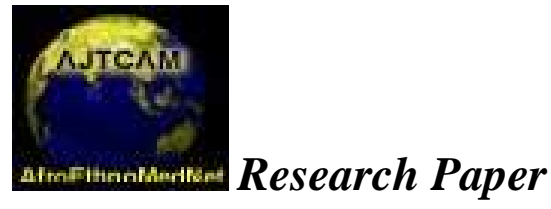

Afr. J. Infect. Diseases

www.africanethnomedicines.net

ISSN: 2006-0165@2008

\title{
COST-EFFECTIVENESS ANALYSIS OF MICROSCOPY, CULTURE AND SENSITIVITY STUDY AS A DECISION TOOL IN CHOICE OF ANTIBIOTICS IN CLINICAL PRACTICE.
}

\author{
*Shaibu O. Bello ${ }^{1,2}$, Simeon A. Isezuo ${ }^{3}$, A. Chika ${ }^{1}$. \\ ${ }^{1}$ Department of Pharmacology, College of Health sciences, Usmanu Danfodiyo University, Sokoto, \\ Nigeria, ${ }^{2}$ Ultrasound and Fertility Unit, Karaye Hospital, Emir Yahaya Road, Sokoto, Nigeria. \\ ${ }^{3}$ Department of Medicine, Usmanu Danfodiyo University Teaching Hospital, Sokoto. \\ *E-mail: bellooricha@yahoo.com
}

\begin{abstract}
This study evaluated the clinical utility and cost effectiveness of traditional MCS studies as decision tools in choosing antibiotics to treat infections in two tertiary hospitals in Northern Nigeria by retrospective evaluation of MCS records over six consecutive months. Of the 942 MCS studies that included 385(40.9\%), 296(31.4\%), 144 $(15.3 \%) 45(4.8 \%), 40(4.3 \%), 26(3.1 \%)$ and $6(0.2 \%)$ urine, high vaginal swabs (HVS), stool, sputum, pus, endocervical swabs (ECS) and urethral swabs specimens performed, respectively on 510(54\%) males and 432 (46\%) females, only $9(0.6 \%)$ had results that offered demonstrable influence on patients' treatment at a cost effectiveness ratio of 4260.6 International dollars per 1. The estimated cost of MCS studies to the Nigerian economy (public health care provider) was 39.8 million International dollars per annum. Our findings suggest that the traditional algorithm for decision on choice of antibiotic is not cost-effective. We proposed that empirical treatment based on community profiling of bacterial sensitivity to antibiotics may be superior.
\end{abstract}

Key words: Traditional MCS studies, Antibiotics, tertiary hospitals, Nigeria

\section{Introduction}

Traditionally, antibiotics are chosen empirically for a suspected infection based on the principle of background probability that, among others, frequently considers the most likely infective agent(s), its known sensitivity profile, availability of the antibiotic, allergy status of the patient, and cost. This choice is usually slated to be updated by the result of a microscopy-culture and sensitivity (MCS) study on an appropriate sample taken before initiating empirical therapy. Though this algorithm has come to be considered as standard of practice, it is probably based on clinical tradition and plausible biology rather than hard evidence of the utility of MCS studies. There are reasons to expect problems with MCS studies. MCS is an in vitro study which may not be transposable to the anticipated in vivo site of action of the antibiotics. When a positive culture is obtained, its clinical utility depends on the appropriateness of the sample, its quality, handling, transportation, laboratory technology, and technologist. These components may fail at any level. These suspicions have been supported by studies evaluating specific infectious illnesses. Several studies have questioned the usefulness of MCS in the management of patients with community-acquired pneumonia (García-Vázquez et al., 2004), respiratory infections (Ewig et al., 2002), pyelonephritis (Wing et al., 2000; Thanassi, 1997), gonorrhea (Ghanem et al., 2004) and gynecological infections (Chandeying et al., 1998). Though some guidelines have cautiously tried to expunge routine MCS studies, it continues to be a tradition recommended by clinicians and policy makers (American Thoracic Society, 2001).To the best of our knowledge, no study has attempted to put a pan-establishment cost effectiveness profile to MCS studies. Such studies are necessary to guide clinical and policy decisions. This study was therefore designed to address the primary questions of the clinical usefulness and cost-effectiveness of MCS study in clinical practice. 


\section{Methods}

We collected retrospective laboratory paper records of consecutive cultures and sensitivity results from two tertiary centers in northwestern Nigeria for six months ( $1^{\text {st }}$ January 2006 to $30^{\text {th }}$ June 2006). The information of interest were hospital identity number (ID), sex, age, type of sample, presence or absence of bacterial growths and the antibiotic sensitivity results. We traced the patients' files using hospital number and referral unit and reviewed the antibiotic treatment post MCS study result. We considered only MCS studies whose result either lead to a recorded change in pre-test antibiotic regimen or fit the pre-test antibiotic regimen and recorded by the clinician as having influenced clinical decision.

Data was analyzed using SYSTAT 12 software. We analyzed MCS data with intention to treat which in this case refers to 'intention to use'. It is thus assumed that all MCS studies were performed with an intention to use the result in decision making. Thus absence of record of use of MCS result, failure of patient to come back to collect the MCS result and failure of patients to come for follow up were all considered as failures. We calculated simple descriptive statistics of parameters of interest and derived the Odds and Risk ratios of all outcomes of MCS studies using SYSTAT 12 Software. We assumed presence or absence of bacterial growth (henceforth referred to only as 'growth') to be independent and calculated the numbers needed to treat which we then translated as 'numbers needed for growth' (NNG) (i.e. any growth irrespective of type) and/or 'numbers needed for potential clinical impact' ( i.e. any growth with associated sensitivity profile, because anecdotal evidence show that sensitivity profiles are not usually done for Candida albicans nor growths considered as normal flora). We also calculated the 'numbers needed for demonstrated clinical impact' (i.e. growths that resulted in a change of antibiotic or resulted in a decision to continue ongoing treatment regimen because of the growth) as additional measures of the clinical utility of MCS.

$* *$ Numbers needed for growth $(N N G)=$ reciprocal of the Risk ratios $(R R)$ of growth $=\{R R \text { (growth) }-R R \text { (no growth) }\}^{-1}$

$* *$ Numbers needed for potential clinical impact $=$ reciprocal of the Risk ratios of having a sensitivity result $=\{$ RR (growth with sensitivity result)-RR (growth without sensitivity result) $\}^{-1}$

**Numbers needed for demonstrated clinical impact= reciprocal of the Risk ratios of using the result of sensitivity study in choosing an antibiotic $=\{R R$ (growth with sensitivity result)-RR (growth with sensitivity result that was used in treatment) $\}^{-1}$

We performed the cost effectiveness analysis as part of sectoral cost effectiveness (David et al., 2006; Pascalina et al., 2007) because we consider MCS to best fit allocation rather than technical efficiency. We evaluated the cost and health benefit of MCS alone with respect to the counterfactual case that it is not in place as an intervention( so called null scenario)(David et al., 2006). We estimated costs using standard costing approach in economic evaluation (David et al., 2006; Pascalina et al., 2007). Costing was done from both providers' and patients' perspectives. Cost elements included were, personnel, materials, building space, administrative and equipment overheads on the one hand, then direct cash cost to patients. We designed and validated questionnaires which were then used to gather data from both primary and secondary sources. The purchase prices were used to derive discounted annual capital cost at a discount rate of 3\% and life span of 5 years (Raymond et al., 2003). We collected salary data from staff members and computed labor costs by evaluating the time spent by each staff cadre in the process of performing an MCS (taking sample, transporting sample, laboratory processes etc) and multiplied this by the pro- rata earnings for each staff category. The direct cash paid by the patient for service was estimated. We did not include costs incurred by patients (and accompanying persons) to access service and cost on lost time and potential earnings in lieu of interventions because it has been accepted that they are not reliably evaluated and are conjectural; they are often excluded from studies on ethical grounds(David et al., 2006; Pascalina et al., 2007). We determined the average cost of MCS in the Nigerian local currency (Naira) and determined its equivalent in International dollars (i.e. the amount of domestic currency that is of equivalent purchasing power as a dollar in the United State of America) (The World Bank, 2007) using the standardized conversion table (International Diabetic Association, 2007) because this has been shown to reflect more comparable cost statements (The World Bank, 2007). The average cost effectiveness ratio (ACER)( David et al., 2006; Pascalina et al., 2007), defined as the total cost of all the MCS studies divided by the numbers of MCS studies with demonstrable contribution to clinical decision was then estimated. The sample studied was considered as the study population in its own right and therefore required neither confidence intervals nor median scores. This is because it is our opinion that it in intuitive that MCS studies would be highly variable both temporal and geometric within and between laboratories, and that this makes attempts at extrapolating inferences to all MCS as the study population ,as would be expected in classical data analysis, at best spurious. Furthermore, it is accepted that cost effectiveness has many uncertainties that cannot be captured by statistical confidence intervals (Pascalina et al., 2007). 


\section{Results}

In the 6 months period reviewed, 942 MCS studies including 510(54\%) on males and $432(46 \%)$ on females were performed. Of the 942 MCS studies, 385 (40.9\%), 296(31.4\%), 144 (15.3\%) 45 (4.8\%), 40 (4.3\%),

Table 1: Average utility of M/C/S studies

$\begin{array}{llll}\text { MCS STUDIES } & \text { NG } & \text { NGPCI } & \text { NGDCI } \\ \text { Urine } & 3 & 175 & 104 \\ \text { HVS } & 2 & 3 & 148 \\ \text { Stool } & 4 & 13 & 72 \\ \text { Sputum } & 9 & 9 & 45 \\ \text { Pus } & 2 & 2 & 40 \\ \text { ECS } & 1 & 1 & \text { Infinite } \\ \text { Urethral } & 2 & 2 & \text { Infinite }\end{array}$

NG: Number of studies needed to get one study with a growth

NGPCI: Number of studies needed to get one study with a growth that had potential clinical impact

NGDCI: Number of studies needed to get one study with a growth that had demonstrable clinical impact

Table 2: Average cost of conducting M/C/S per patient

$\begin{array}{llll}\text { Item Number } & \text { Cost Item } & \text { Cost } \text { (Int. \$) } & \text { Percentage of Total Cost } \\ 1 & \text { Consumable Materials } & 12.0 & 44.28 \\ 2 & \text { Personnel } & 5.1 & 18.82 \\ 3 & \text { Building Space } & 2.1 & 7.75 \\ 4 & \text { Administrative and } & 1.5 & 5.54 \\ & \text { other overheads } & & \\ 5 & \text { Equipment and building } & 2.4 & 8.86 \\ 6 & \text { Cost to patient } & 4.00 & 14.76 \\ \mathbf{7} & \text { Average total cost per } & \mathbf{2 7 . 1} & \mathbf{1 0 0} \\ & \text { M/C/S } & & \end{array}$

Table 3: Average cost effectiveness of $\mathrm{M} / \mathrm{C} / \mathrm{S}$

Cost

To The Patient

Total

27.1

*ACER does not obey mathematical addition down a column because the denominator varies between provider and patient

$26(3.1 \%)$ and $6(0.2 \%)$ were on urine, high vaginal swabs (HVS), stool, sputum, pus, endocervical swabs (ECS) and urethral swabs, respectively. Of the whole sample, $603(64 \%)$ had no significant growth (Odds of no growth: 178: 1), while 339(36\%) had growth; (Odds of growth: 56: 1). The odds ratio of no growth to growth from the specimens was 3.2). Of the 339 cultures with growth, 37 (11\%) provided no additional information of clinical relevance (Candiasis), and 302(89\%) had sensitivity profiles. The Odd of no additional information of clinical relevance from MCS with growths was: 213: 1 and Odd of additional information of clinical relevance was 47: 1(Odds ratio of no additional information of clinical relevance to getting additional information of clinical relevance was 4.5).

Only $9(0.6 \%)$ of the 942 MCS studies either fit the current treatment regimen or lead to a change in antibiotic therapy of the subjects. The Odd of an MCS being of clinical utility was 0.0097 . Thirteen MCS studies 
would have been performed to have 1 MCS with potential impact on clinical decision while 105 MCS studies were required to have 1 with demonstrable clinical impact. Of the 385 urine MCS, 314(81.3\%) were on males, of which $274(87.3)$ were aged above 20 years and $40(12.7 \%)$ were all under 10 years. $71(18.7 \%)$ were on females, of which $57(80.3 \%)$ were aged above 20 years, $7(9.9 \%)$ were aged 14-20yrs and 7(9.9\%) aged 13 years and below. There was no growth in $269(69.8 \%)$ samples while 116(30.2\%) had growths.

Fifty two $(87.3 \%)$ of the female urine studies had no growth while $217(69.1 \%)$ of the male studies had no growth. The number of urine studies needed to obtain a growth was 1 in 3 but the number needed for demonstrable clinical impact was 1 in 104 in males and 1 in 71 in females (Table 1). Of the 296 HVS MCS studies, 99 (33.4\%) had no growth and 197(66.6\%) had growth including Candida Albicans 33(16.7\%), normal flora 60(30.5\%) coliforms $104(52.8 \%)$. The numbers needed for a growth was 1 in 2 studies, while the numbers needed to have a growth of potential clinical impact was 1 in 3 studies. The numbers needed for demonstrated clinical impact was 1 in 148 studies. Of the $26 \mathrm{ECS}, 11(42.3 \%)$ had no growth and $15(57.7 \%)$ had growth of which none contributed to clinical decision. Of the 144 stool studies, $110(76.4 \%)$ had no growths and $34(23.6 \%)$ had growths. The number needed for a growth was 1 in 4, the number needed for a growth of potential clinical benefit was 1 in 13 and numbers needed for demonstrated clinical benefit was 1 in 72 . Of the 45 sputum, 20(44.4\%) had no growth and $25(55.6 \%)$ had growth. The numbers needed for growth with demonstrated clinical impact was 1 in 45 . Of the 40 pus studies 19 $(47.5 \%)$ had no growth and $21(52.5 \%)$ had growth. Numbers needed for growth and numbers needed for growth with potential clinical impact were both 1 in 2 . Numbers needed for growth of demonstrable clinical impact was 1 in 40 . Of the 6 urethral swabs, 2(33.3\%) had no growth while 4 (66.7\%) had growths. Numbers needed for growth and numbers needed for growth with potential clinical impact were both 1 in 2 . In none of the growths was there demonstrable contribution to clinical decision.

The average cost of one MCS was 23.1 International dollars to the provider and 4.0 International dollars to the patient. The ACER of MCS was 4,260.6 International dollars to 1. Estimated annual cost of MCS was 51,127.6 International dollars per hospital setting. There is at least 1 General hospital per Local Government Council in Nigeria. The annual cost of MCS studies for the 774 councils in Nigeria was 39.8 million International dollars. . Test specific ACER was 4,016.4 International dollars to 1 for HVS, 2,822.3 International dollars to 1 for Urine studies in males, 1,926.8 International dollars to 1 for urine studies in females, 1,953.9 International dollars to 1 for ECS, 1,221.2 International dollars to 1 for sputum studies, 1,085.6 International dollars to 1 for pus studies and indeterminate for urethral swab.

\section{Discussion and Conclusion.}

MCS studies have been built and accepted 'Volta face' into traditional clinical protocol on the reasonable assumption that it would be of clinical utility. To the best of our knowledge, this study is the first one that has attempted to evaluate this assumption. There was no significant difference between number of males and females referred for MCS studies. The finding that the triad of urine, high vaginal and stool studies constitutes over $85 \%$ of MCS studies is expected in a third world setting where sexually transmitted and gastrointestinal infections are leading causes of morbidities and those with which clinicians may have higher concerns about antibiotic failures leading to a high rate of request for MCS. The finding that $64 \%$ of MCS studies had no bacterial growth should ordinarily suggest that, within the limits of bacteria like Mycoplasma and Chlamydia with special needs for culture, no bacterial infection exists at the site from which specimens were obtained, and probably suggests withdrawal of antibiotics. If so, clinical utility could be claimed. As it stands it remains that based on our intention to use model, $64 \%$ of MCS studies have failed because absence of growth was not found to have been utilized to guide antibiotic usage.

That only $0.6 \%$ of MCS studies translated into decisions on antibiotic therapy appears incredulous but leading faculties in the study zone are not surprised as it fits into their overall general experience (Ekele BA, Legbo JN, Usmanu Danfodiyo University, personal communications). Given that clinicians would not be comfortable with choices made by tossing of a coin despite the odds of its outcome being 1 in 2 tosses, the 178:1 odd of not obtaining a growth from MCS is extremely discouraging. This observation is compounded by both the infinitesimal probability of 0.0097 that any MCS study is of clinical utility and that 105 MCS have to be performed for 1 study to have demonstrable clinical impact. The wide gap between the number of MCS studies needed for one MCS to have potential impact on clinical decision (1 in 13) and numbers needed for demonstrable clinical impact (1 in 105) may suggest attrition in utility between laboratory output and clinicians but this finding needs further evidence based explanation. The results of the MCS-type specific analysis did not show significantly improved measures of clinical utility except for urine MCS in females that has a number needed for clinical utility of 1 in 71 . The ACER of 
4,260.6 International dollars for any-MCS study is discouraging. This study was not designed to compare test specific ACER and such comparison may not be valid post-hoc because comparative samples sizes were not used. What is the acceptable cost of a useful MCS study? If only 9 in 942 studies were clinically useful but saved life, would it be considered cost effective to expend 51,127.6 international dollars per annum to save at least 1 life? Also, wouldn't it be considered cost effective if one HVS study prevented pelvic infection, tubal blockage and infertility when compared against the cost of treating infertility? Our data do not address these questions. The ACERs are, therefore, best taken as stand alone assessments. Given this, none of the MCS studies appear cost effective on face value.

An alternative to MCS studies may be desirable. This study suggests that empirical antibiotic therapy based on literature-based listing of so called 'drugs -of -choice' is probably successful, and perhaps more so than patient based MCS studies. It, therefore, appears plausible that sensitivity profiling of bacterial species within defined geographical zones may outperform even literature based profiling.

An obvious shortcoming of this study is that the observations may only be true at the study points and that laboratory services may be on the weak side. If so, this study may then actually represent an audit that suggests system failure. There is no evidence, beyond profiling of third world medical services, to support such a deduction. Moreover, MCS studies should be basic procedures in any tertiary centre. Studies in advanced countries showing yields of $2.8-5 \%$ on culture specimens supports our findings (Meropol et al., 1997). There is no denying that a system analysis may be needed to find out the reason for the poor cost effectiveness parameters of MCS and subsequently correct them. However, it will still require re-evaluation to demonstrate that such changes do lead to improvement in performance. Even if the shortcomings are taken as true, the result of this study still emphasizes the need for systematic evaluation of clinical algorithms whose effectiveness appears obvious in order to obtain hard evidence that it is indeed so. Given the huge cost implication, a repeat of similar assessment of MCS studies at all treatment points may be desirable with the aim of expunging it from clinical protocols if confirmed.

\section{References}

1. García-Vázquez, E., Marcos, M. A., Mensa, J., de Roux, A., Puig, J., Font, C., Francisco, G., Torres, A. (2004). Assessment of the Usefulness of Sputum Culture for Diagnosis of Community-Acquired Pneumonia Using the PORT Predictive Scoring System. Arch Intern Med. 164:1807-1811

2. Ewig, S., Schlochtermeier, M., Göke, N. and Niederman, M. S. (2002). Applying Sputum as a Diagnostic Tool in Pneumonia: Limited Yield, Minimal Impact on Treatment Decisions. Chest, 121:1486-1492

3. Wing, D. A., Park, A. S., DeBuque, L.\$. and Millar, L. K. (2000). Limited clinical utility of blood and urine cultures in the treatment of acute pyelonephritis during pregnancy. Am J Obstet Gynecol.,182:1437-1440

4. Thanassi, M. (1997). Utility of urine and blood cultures in pyelonephritis. Acad Emerg Med ., 4:797-800

5. Ghanem, M., Radcliffe, K. and Allan, P . (2004). The role of urethral samples in the diagnosis of gonorrhea in women. International Journal of STD \& AIDS, 15: 45-47

6. Chandeying, V., Skov, S., Kemapunmanus, M., Law, M., Geater, A. and Rowe, P. (1998). Evaluation of two clinical protocols for the management of women with vaginal discharge in southern Thailand. Sex. Transm. Inf. 74:194-201.

7. American Thoracic Society (2001). Guidelines for the management of adults with community-acquired pneumonia: diagnosis, assessment of severity, antimicrobial therapy, and prevention. Am J Respir Crit Care Med.163:1730-1754

8. David B. E., Tessa T., Taghreed A. and Stephen S. L. (2006). Choosing Interventions that are Cost Effective (CHOICE) Millennium Development Goals Team. Methods to assess the costs and health effects of interventions for improving health in developing countries. BMJ 331:1137-1140

9. Pascalina, C., Felix, M., Bona, M. C., Naawa, S., Moonga, H., Patrick, B. and Okorosobo, T. (2007). A costeffectiveness analysis of artemether lumefantrine for treatment of uncomplicated malaria in Zambia. Malaria J. 6:21 .

10. Raymond H., Dan C., Tessa T. E. and WHOCHOICE (2003). Generalized cost-effectiveness analysis for nationallevel Priority-setting in the health sector. Cost Effectiveness and Resource Allocation 2003; 1:8

11. The World Bank. (2007). http://web.worldbank.org/WBSITE/EXTERNAL/DATASTATISTICS/0,,contentMDK:20399244 menuPK:1504474 pagePK:64133150 piPK:64133175 theSitePK:239419,00.html . Accessed 15/07/07

12. International diabetic federation (2007). http://www.eatlas.idf.org/Costs_of_diabetes/Calculated_cost_estimates/International_dollar Accessed 15/07/07

13. Meropol, S.B., Luberti, A.A., De jong, A.R. (1997). Yield from stool testing of paediatric in patients. Arch Paediatr Adolesc Med. 151:142-145" 